\title{
An effective method for trapping ion beams in superfluid helium for laser spectroscopy experiments
}

\author{
X.F Yang ${ }^{1,2, a}$, T. Furukawa ${ }^{3,2}$, T.Wakui ${ }^{4}$, K. Imamura ${ }^{6,2}$, H. Fujita ${ }^{5}$, Y. Mitsuya ${ }^{6}$, M. Hayasaka $^{7}$, \\ Y. Ichikawa ${ }^{8}$, Y. Ishibashi ${ }^{9,2}$, H. Shirai ${ }^{8}$, Y. Ebara $^{3}$, A. Hatakeyama ${ }^{10}$, M. Wada ${ }^{2}$, T. Sonoda $^{2}$, \\ T. Ito ${ }^{2}$, T. Kobayashi ${ }^{11}$, S. Nishimura ${ }^{2}$, M. Nishimura ${ }^{2}$, Y. Kondo ${ }^{2}$, K. Yoneda ${ }^{2}$, H. Ueno ${ }^{2}$, T. \\ Shinozuka ${ }^{4}$, T. Shimoda $^{5}$, K. Asahi ${ }^{8}$, and Y. Matsuo ${ }^{2}$ \\ ${ }^{1}$ School of Physics, Peking University, Chengfu Road, Haidian District, Beijing, 100871, China \\ ${ }^{2}$ RIKEN Nishina Center, 2-1 Hirosawa, Wako, Saitama 351-0198, Japan \\ ${ }^{3}$ Dept. of Physics, Tokyo Metropolitan University, 1-1 Minami-Osawa, Hachioji, Tokyo 192-0397, Japan \\ ${ }^{4}$ Cyclotron and Radioisotope Center, Tohoku University, 6-3 Aoba, Aramaki, Aoba, Sendai, Miyagi, 980- \\ 8578, Japan \\ ${ }^{5}$ Dept. of Physics, Osaka University, 1-1 Machikaneyama, Toyonaka, Osaka 560-0043, Japan \\ ${ }^{6}$ Dept. of Physics, Meiji University, 1-1-1 Higashi-Mita, Tama, Kawasaki, Kanagawa 214-8571, Japan \\ ${ }^{7}$ Dept. of Physics, Tokyo Gakugei University, 4-1-1 Nukuikitamachi, Koganei, Tokyo 184-8501, Japa \\ ${ }^{8}$ Dept. of Physics, Tokyo Institute of Technology, 2-12-1 Ookayama, Meguro, Tokyo 152-8551, Japan \\ ${ }^{9}$ Dept. of Physics, university of Tsukuba, 1-1-1 Tennodai, Tsukuba, Ibaraki 305-8571, Japan \\ ${ }^{10}$ Dept. of Applied Physics, Tokyo University of Agriculture and Technology, 2-24-16 Naka-cho, Koganei, \\ Tokyo 184-8588, Japan \\ ${ }^{11}$ RIKEN Advanced Science Institute, 2-1 Hirosawa, Wako, Saitama 351-0198, Japan
}

\begin{abstract}
A novel laser spectroscopy technique -"OROCHI" (Optical Radioisotopes Observation in Condensed Helium as Ion-catcher) has been proposed. This method aimed to investigate the structure of exotic nuclei systematically by measuring nuclear spins and moments. For in-situ laser spectroscopy of atoms in He II, a method to trap atoms precisely at the observation region of laser is highly needed. In this work, a setup composed of a degrader, two plastic scintillators and a photon detection system is further tested and verified for adjusting and checking the stopping position of ${ }^{84-87} \mathrm{Rb}$ beam. Details of the current setup, experimental results using this method are presented.
\end{abstract}

\section{Introduction}

To study the nuclear structure of unstable nuclei by measuring properties of nuclei ground and isomeric state such as nuclear spins and moments [1], a novel laser spectroscopic technique for radioisotope (RI) atoms-"OROCHI" (Optical Radioisotopes Observation in Condensed Helium as Ion-catcher) has been proposed in recent years. This technique, combining dense superfluid helium (He II) for trapping energetic RI beams easily during its lifetime [2] and in-situ nuclear laser spectroscopy of stopped RI atoms, has potential to overcome experimental difficulties for measuring the nuclear structure of unstable nuclei with low yield and high contamination. OROCHI method takes advantage of the characteristic optical properties of atoms in He II [3] and atomic laser physics methods such as optical detection, optical pumping and double resonance. Therefore, it is supposed to be feasible to measure the spins, electromagnetic moments in ground and isomer state RIs of a wide variety of atomic species

ae-mail: yangxf@ ribf.riken.jp 
with extremely low yield. Currently, the OROCHI technique is developing and improving with the offline and online experimental research of stable atoms [4-6].

For in-situ laser spectroscopy of atom immersed in He II, trapping RI beam at observation region with laser irradiation is crucial. Therefore, an effective method for adjusting and observing the stopping position of energetic RI beam in He II has been demonstrated with the primary ${ }^{85,87} \mathrm{Rb}$ and secondary ${ }^{84,86} \mathrm{Rb}$ beams. In this paper, we will present the setup, working procedure and results of the experiment for this method. The calculation results with LISE++ program [7] using Zeigler code [8] are also reported for a prior estimation and a comparison with experimental result.

\section{Experimental setup}

For OROCHI experiments performed at RIKEN Projectile fragment Separator (RIPS) for ${ }^{84-87} \mathrm{Rb}$, a combination of Al degrader, plastic scintillators in both the front and the center of cryostat, and a photon detection system placed under the cryostat has been used to stop and trap the beam (66 Mev/u for primary ${ }^{85,87} \mathrm{Rb}$ and $\sim 62 \mathrm{MeV} / \mathrm{u}$ for secondary beam ${ }^{84,86} \mathrm{Rb}$ ) efficiently at the precise position of He II, as shown in Fig. 1. Adjusting of RI beams is achieved by the energy degrader made of aluminum foils, which is the common technique used for trapping of particles $[9,10]$. These aluminum foils are mounted on two separate rotary wheels and their thickness differs from their neighbor by $12.5 \mu \mathrm{m}$ and $100 \mu \mathrm{m}$ for two wheels, respectively. By rotating two wheels for choosing the various combination of foils, which are remotely controlled with PC, the total thickness of degrader can be adjusted from 0 to $787.5 \mu \mathrm{m}$. The $12.5 \mu \mathrm{m}$ difference of the degrader thickness causes the difference of stopping position as less than $0.2 \mathrm{~mm}$, which is sufficiently suitable for the required precision in our current system (66 $\mathrm{MeV} / \mathrm{u}$ primary Rb beam). One plastic scintillator (Tr-PL, Saint Gobain Co.Ltd., BC 408, thickness: $100 \mu \mathrm{m}$ ) assembled with two photomultiplier tubes (Hamamatsu Photonics Co.Ltd., H6533) placed in a pre-cryostat chamber is used to count the ions introduced into the cryostat chamber. Another scintillator (He-PL, Saint Gobain K. K., BC 408, thickness: $500 \mu \mathrm{m}$ ) located at the center of the cryostat is used to check the stopping position of atoms by photon counting using photon detection system. He-PL can be removed from the center of cryostat without stopping the cryostat operation. The photon detection system is mainly used to count laser induced fluorescence (LIF) photons from the trapped atoms optically pumped by the laser beam (cw Ti:Sapphire laser, 899-01, Coherent Co.Ltd.). It is composed of three large Fresnel lenses, a pair of orthometric slits remotely controlled with PC,

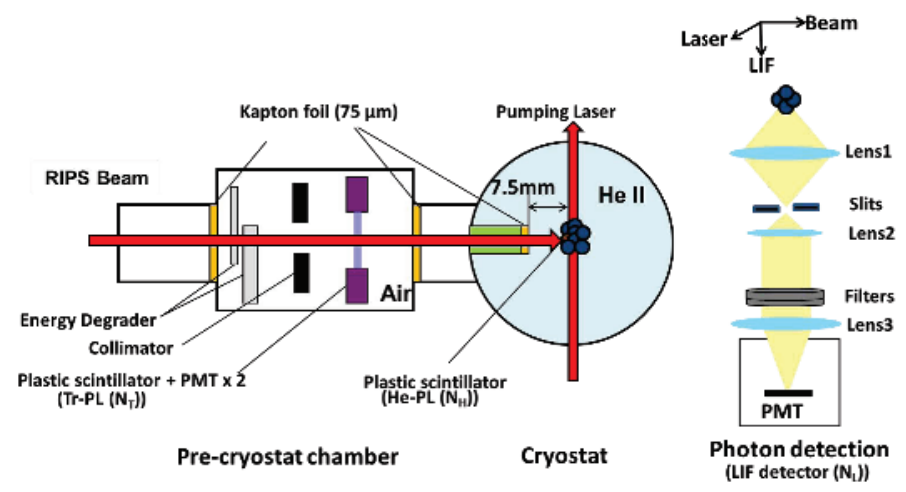

Figure 1: A schematic of the current system for checking stopping position of beam in He II. Al degraders, plastic scintillators in both the front and center of cryostat and a photon detection system placed under the cryostat are used to stop the beam in He II. The photon counts from Tr-PL, He-PL and laser induced fluorescence (LIF) are marked by $\mathrm{N}_{T}, \mathrm{~N}_{H}$ and $\mathrm{N}_{L}$, respectively, in the text. 
INPC 2013

Table 1: The optimal degrader thickness for a stopping position at exactly $7.5 \mathrm{~mm}$ is listed for both LISE++ calculation results for ${ }^{84-87} \mathrm{Rb}$ with Ziegler program code and OROCHI experimental results with primary ${ }^{85,87} \mathrm{Rb}(66 \mathrm{MeV} / \mathrm{u})$ and secondary beam ${ }^{84,86} \mathrm{Rb}(\sim 62 \mathrm{MeV} / \mathrm{u})$.

\begin{tabular}{|c|c|c|c|c|}
\hline & ${ }^{87} \mathrm{Rb}$ & ${ }^{86} \mathrm{Rb}$ & ${ }^{85} \mathrm{Rb}$ & ${ }^{84} \mathrm{Rb}$ \\
\hline Calculated results & $344.0( \pm 1.8) \mu \mathrm{m}$ & $59.0( \pm 15.0) \mu \mathrm{m}$ & $307.5( \pm 1.8) \mu \mathrm{m}$ & $70.1( \pm 15.6) \mu \mathrm{m}$ \\
\hline Experimental results & $350.0( \pm 6.3) \mu \mathrm{m}$ & $62.5( \pm 6.3) \mu \mathrm{m}$ & $337.5( \pm 6.3) \mu \mathrm{m}$ & $50.0( \pm 6.3) \mu \mathrm{m}$ \\
\hline
\end{tabular}

band-pass and edge-pass interference filters at both He-PL photon and LIF wavelength, and a cooled photomultiplier tube (Hamamatsu Photonics Co.Ltd., R943-02 and C10372). The volume of the observation region for the photon detection system is determined by adjusting the slits width and the laser diameter. For experiment with ${ }^{84-87} \mathrm{Rb}$ beam, we used the volume $5 \times 2 \times 2 \mathrm{~mm}^{3}$ by fixing the slits at $5 \mathrm{~mm}$ along the laser path and $2 \mathrm{~mm}$ along the beam direction with a laser of $2 \mathrm{~mm}$ diameter.

\section{Calculated and experimental results}

Calculation using LISE++ program are needed prior to the experiment in order to estimated the stopping position of beam in He II and to compare with the experimental results. The Ziegler code which is appropriate for calculating energy loss of ions in matter at low and intermediate energies was used for the calculation of stopping position of ${ }^{84-87} \mathrm{Rb}$ isotopes in He II. Calculated degrader thickness for stopping position at $7.5 \mathrm{~mm}$ from the entrance window in He II are listed in Table 1. The straggling (FWHM) of stopping position of secondary beam ${ }^{84,86} \mathrm{Rb}$ in calculation is about $0.5 \mathrm{~mm}$ corresponding to approximately $30 \mu \mathrm{m}$ straggle of degrader thickness, which comes from large energy spread of secondary beam produced by the projectile-fragment reaction of primary beam (energy: $66 \mathrm{MeV} / \mathrm{u}$ ).

In OROCHI experiments, we have successfully trapped the beams ${ }^{84-87} \mathrm{Rb}$ at an accurate stopping position in He II for further laser spectroscopy experiment. The actual procedure for determine the stopping position of the beam is as follows (using ${ }^{87} \mathrm{Rb}$ as an example): First, from the prior LISE++ calculation using Ziegler's program code, we estimated an appropriate degrader thickness as 344.0 $\mu \mathrm{m}$ for stopping the ${ }^{87} \mathrm{Rb}$ at the center of He II (Table 1), where the beam path is $7.5 \mathrm{~mm}$ between the entrance window and the surface of He-PL. Subsequently, during beam time, we decreased the incident beam energy step by step using the degrader to stop the beam near the surface of He-PL which is checked by the count ratio of He-PL to Tr-PL. From the measured relation between the ratio $\mathrm{N}_{H} / \mathrm{N}_{T}$ and the degrader thickness, we determined the optimum degrader thickness fell in the range of $350.0 \sim 400.0 \mu \mathrm{m}$ for ${ }^{87} \mathrm{Rb}$ (Fig. 2(a)) where the ratio tends towards minimum. After removing the He-PL from the laser observation region, we pass through laser from the center of He II. The overlap of stopped atoms with the laser beam is then confirmed by the detected LIF intensity from the stopped atoms optically pumped by laser beam in He II. The maximum ratio $\mathrm{N}_{L} / \mathrm{N}_{T}(350 \mu \mathrm{m}$ for ${ }^{87} \mathrm{Rb}$ in Fig. 2(b)) corresponds to the best overlap between the region where implanted atoms stopped and trapped, which suggests that the accelerated atoms are stopped at the correct position. The same procedure was also performed for stopping the isotope of ${ }^{84,85,86} \mathrm{Rb}$ in He II. All of the experimental results are shown in Fig. 2, and listed in Table 1. The results satisfy required accuracy for further laser spectroscopy experiment, which can be confirmed from the consistency of the straggling of the stopping position (about $1.5 \sim 2 \mathrm{~mm}$ deduced from the $50 \mu \mathrm{m} \sim 75 \mu \mathrm{m}$ width of Gaussian fitting curve in Fig 2(b) and (c)) and the diameter of laser beam (about $1.5 \sim 2 \mathrm{~mm}$ ). Recently we perform the OROCHI experiment successfully by use of the precise determination of the stopping position of introduced beams. Both the polarization and double resonance signal of the atoms immersed in He II have been observed successfully, part of which has been presented in [11].

In order to confirm the reliability of the LISE++ calculation with the Ziegler code, the calculated results of the energy loss in $\mathrm{He}-\mathrm{Pl}$ of primary ${ }^{85,87} \mathrm{Rb}$ beam with different degrader thickness are also 




(a)

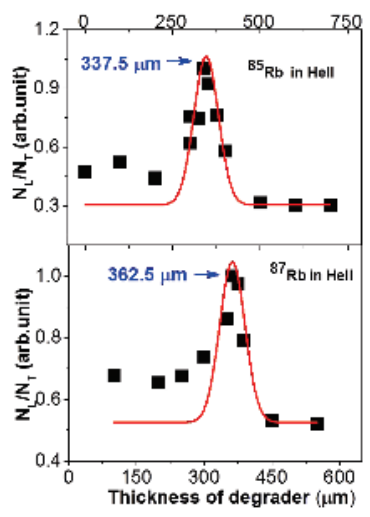

(b)

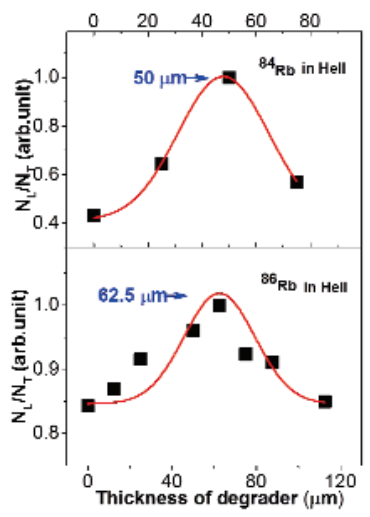

(c)

Figure 2: Experimental results for ${ }^{84-87} \mathrm{Rb}$ in He II. (a): Relation between the ratio $\mathrm{N}_{H} / \mathrm{N}_{T}$ and degrader thickness for ${ }^{85-87} \mathrm{Rb}$ with the LISE++ calculation of the energy loss of primary ${ }^{85,87} \mathrm{Rb}$ beam in He-PL. (b) and (c): The ratio $\mathrm{N}_{L} / \mathrm{N}_{T}$ as a function of degrader thickness for ${ }^{84-87} \mathrm{Rb}$. All of the $\mathrm{Y}$ axis is normalized with the maximum ratio of counts.

plotted in Fig. 2(a) together with the experiment results as a comparison. A good consistent can be found for the results, which can also be confirmed from Table 1.

\section{Summary}

The feasibility of the method aimed to trap energetic RI at an accurate position in He II was confirmed by the intercomparison of the LISE++ calculation and the experimental results with ${ }^{84-87} \mathrm{Rb}$ beam. This gives us confidence in conducting future OROCHI experiment for nuclear laser spectroscopy. We now continue to develop and improve the method to control and confirm the stopping position of beam in He II precisely, for laser spectroscopy of a wide range of atomic species in different energy regions in He II.

\section{References}

[1] B. Cheal, et al., Journal of Physics G: Nuclear and Particle Physics 37 (2010) 113101.

[2] T. Shimoda, et al., Nuclear Physics A 588 (1995) c235 - c239.

[3] B.Tabbert, et al., J. Low Temp. Phys. 109 (1997) 653-707.

[4] T. Furukawa, et al., Phys. Rev. Lett. 96 (2006) 095301.

[5] T. Furukawa, et al., Physica E: 43 (2011) 843 - 846.

[6] T. Furukawa, et al., Journal of Physics: Conference Series 302 (2011) 012054.

[7] O. Tarasov, et al., Nucl. Instr. Meth. B 266 (2008) 4657.

[8] J. F. Ziegler, et al., SRIM-The Stopping and Range of Ions in Matter, 2008.

[9] M. H. Holzscheiter, Physica Scripta 46 (1992) 272.

[10] M. Holzscheiter, et al., Nuclear Physics A 558 (1993) 709 - 718.

[11] T. Furukawa, et al., Nucl. Instr. Meth. B 317 (2013) 590. 